\title{
Automatic Generation of Client-Server Collaborative Web Applications from Diagrams
}

\author{
Mitsuhisa Taguchi and Takehiro Tokuda \\ Department of Computer Science, Tokyo Institute of Technology \\ Meguro, Tokyo 152-8552, Japan \\ \{mtaguchi, tokuda\}@tt.cs.titech.ac.jp
}

\begin{abstract}
We have designed and implemented Web application generators based on a diagram approach, which is an approach to generating data-intensive Web applications by using diagrams that represent dataflow relationships among Web components. In this paper, we illustrate how to extend the generator systems to generate client-server collaborative Web applications, in which client-side programs collaborate with server-side programs in performing the whole business logic. The clientside programs contribute toward not only reducing the load on Web servers but improving security and session management of the generated application.
\end{abstract}

\section{Introduction}

We have designed and implemented Web application generators to support development of consistent and secure data-intensive Web applications based on a diagram approach [1-4], in which we first compose diagrams to describe overall behavior of the application, select appropriate programs from predefined and general-purpose ones, and then generate an implementation. Although the current implementation focuses on generating server-side programs, the approach is sufficiently flexible to generate client-server collaborative Web applications, in which client-side programs collaborate with server-side programs in performing the whole business logic. In this paper, we illustrate how to generate client-server collaborative Web applications based on the diagram approach. The extended generator systems can generate both traditional Web applications and clientserver collaborative Web applications from the same diagrams.

\section{Client-Server Collaborative Web Applications}

As services on the Web become complex, we need client-side programs that are activated by client-side events and collaborate with server-side programs in performing the whole business logic. In this paper, we call such Web applications client-server collaborative Web applications. In general, the behavior of the application is as follows (Fig. 1). 


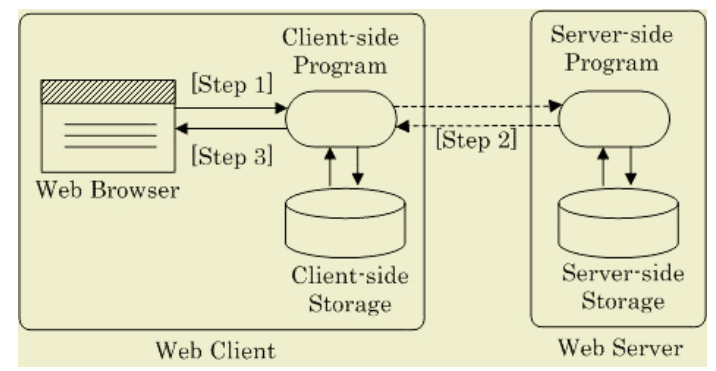

Fig. 1. The architecture of client-server collaborative Web applications

1. A Web client first gets a Web document with client-side programs from Web servers. When the client-side programs are activated, the programs may check the query and change the client-side state.

2. The programs then decide whether a request to a Web server is necessary or not. If there are available data on the client side, the programs process the local data before sending a request.

3. After obtaining the data from the Web server, the client-side programs arrange a Web page to be generated. In addition, the programs may change the client-side state according to the result of the processing.

Client-side programs are expected to contribute toward not only reducing the load on Web servers but improving security and session management of the application. For example, the client-side programs can control the view of Web documents, process the received data before generating a Web page, handle the local data, and manage the client-side state.

\section{Extension of T-Web System}

T-Web system is a system to generate executable data-intensive Web applications from Web transition diagrams, which are special diagrams to describe the behavior of target applications. The nodes and links of the diagrams are (a) a fixed Web page node, (b) an output Web page node, (c) a processing node, (d) a database node, (e) a page transition link, and (f) a data-flow link. The T-Web system has predefined and general-purpose program templates so that it can generate executable Web applications from the diagrams.

Fig. 2 shows an example of the Web transition diagrams and the generation rules for data filtering applications. If we generate the application as a clientserver collaborative Web application, the client-side programs interrupt clients' events. When users try to browse a next page or sort the items, the client-side programs process the catalog data that are downloaded on the client side and then embed the result into the Web page template. The client-side programs can be implemented by Java Applet and JavaScript. For each processing node whose processing can be done on the client side, source code of a client-side program 


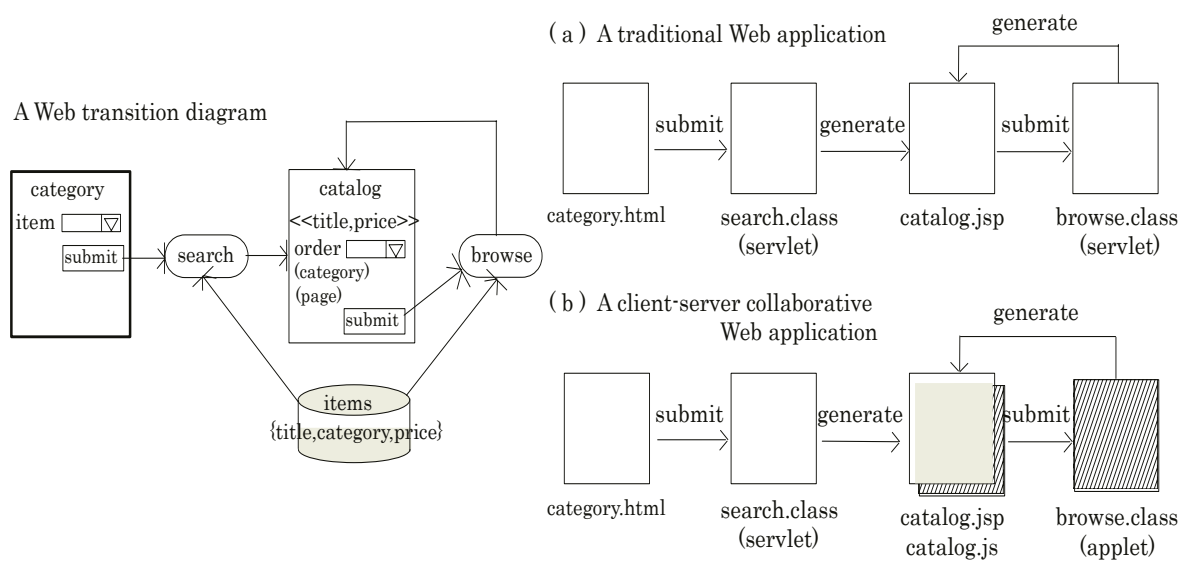

Fig. 2. A Web transition diagram for a Web application with data filtering

such as an applet program is generated. For each fixed/output Web page node whose events activate the above client-side program, a static/dynamic Web page document and a client-side script such as JavaScript are generated.

\section{Conclusion}

In this paper, we illustrated how to generate client-server collaborative Web applications based on a diagram approach. The extended T-Web system can generate both traditional Web applications and client-server collaborative Web applications from the same diagrams called Web transition diagrams. As future work, we may concentrate on generating a program that dynamically measures the capability of Web clients and invokes appropriate programs, together with programs for a variety of Web clients.

\section{References}

1. T. Matsuzaki, T. Suzuki and T. Tokuda. A Pipe/Filter Architecture Based Software Generator PF-Web for Constructing Web Applications. Computer Software of Japan Society for Software Science and Technology Vol.19 No.4, pp.266-282, 2002.

2. K. Jamroendararasame, T. Matsuzaki, T. Suzuki and T. Tokuda. Generation of Secure Web Applications from Web Transition Diagrams. Proc. of the IASTED International Symposia Applied Informatics, pp.496-501, 2001.

3. M. Taguchi, T. Suzuki and T. Tokuda. A Visual Approach for Generating Server Page Type Web Applications Based on Template Method. Proc. of the 2003 IEEE Symposium on Visual and Multimedia Software Engineering, pp.248-250, 2003.

4. M. Taguchi, K. Jamroendararasame, K. Asami and T. Tokuda. Comparison of Two Approaches for Automatic Construction of Web Applications: Annotation Approach and Diagram Approach. Proc. of the 4th International Conference on Web Engineering, pp.230-243, 2004. 